\title{
Numerical Analysis of Rapidly Growing, Nonphotochromogenic Mycobacteria, Including Mycobacterium agri (Tsukamura 1972) Tsukamura sp. nov., nom. rev.

\author{
MICHIO TSUKAMURA
} \\ The National Chubu Hospital, Obu, Aichi, Japan 474
}

\begin{abstract}
Numerical analyses of 165 strains of rapidly growing, nonphotochromogenic mycobacteria were carried out with 104 characters in one case and 84 in another. The results showed that the following taxa are distinct: $M y c o b a c t e r i u m$ fortuitum; $M$. chelonei subsp. chelone $; M$. chelonei subsp. abscessus; $M$. smegmatis; $M$. chitae; and "M. agri" (names in quotation marks are not on the Approved Lists of Bacterial Names, 1980). The distinctness of " $M$. agri" was confirmed by using 29 test strains. Strains of $M$. smegmatis were divided into two subgroups, " $M$. smegmatis subsp. smegmatis" and " $M$. smegmatis subsp. lacticola" on the basis of amidase patterns. Clusters of $M$. fortuitum, $M$. chelonei subsp. chelonei, and $M$. chelonei subsp. abscessus were distinguished at a level of $89 \%$ similarity by a numerical taxonomy method in which 84 characters were used, and these three clusters were connected to each other to form a large cluster at a level of $88 \%$. Therefore, these three taxa were regarded as belonging to a complex. Within the cluster $M$. fortuitum, four subclusters were observed, but about one-half of the $M$. fortuitum strains studied did not belong to any of the four subclusters. The 78 strains of $M$. fortuitum studied were regarded as homogeneous, even though several biovars could be differentiated. Neither subcluster nor biovar of the $M$. fortuitum strains was shown to be significantly associated with disease in humans. Because Mycobacterium agri was not included on the Approved Lists of Bacterial Names, it is herein proposed as a revived name. The type strain of $M$. agri is 90012 (= ATCC 27406).
\end{abstract}

The number of species of rapidly growing, nonphotochromogenic mycobacteria is small. At present, the following species are generally accepted (13, 17-19): Mycobacterium smegmatis (Trevisan) Lehmann and Neumann 1899 (14); $M$. fortuitum Da Costa Cruz 1938 (7); $M$. chelonei Bergey et al. 1923 (1); $M$. chitae Tsukamura 1967 (27); M. farcinogenes Chamoiseau 1979 (6); and Mycobacterium senegalense (Chamoiseau) Chamoiseau 1979 (6). In 1972, Kubica et al. (13) created two subspecies of $M$. chelonei-M. chelonei supsp. chelonei and $M$. chelonei subsp. abscessus-having reduced "Mycobacterium abscessus Moore and Frerichs 1953" (15) (all names in quotation marks are not on the Approved Lists of Bacterial Names [19]) to subspecies rank. Runyon et al. (17) recognized only three species of these mycobacteria: $M$. smegmatis, $M$. chelonei, and $M$. fortuitum. Subsequently, Saito et al. (18), reporting as a study by the International Working Group for Mycobacterial Taxonomy, considered $\boldsymbol{M}$. chitae as an acceptable species. In addition, in 1972, Tsuka- mura (30) proposed "Mycobacterium agri" as a new species. However, this name was not included on the Approved Lists of Bacterial Names (19), and thus one of the objectives of this paper is to effect its valid publication.

To date, all known rapidly growing, nonphotochromogenic mycobacteria have been found in the environment (soil, dust, etc.) (36). Of these, the most commonly occurring is $M$. fortuitum, and only $M$. fortuitum and $M$. chelonei are regarded as opportunistic pathogens (35). The fact that the most commonly occurring organism is an opportunistic pathogen prompts the question of whether some subgroups of this organism are likely to be pathogenic. Bönicke (4) reported three biovars, and Tsukamura et al. (40) differentiated two subspecies, " $M$. fortuitum subsp. fortuitum" and " $M$. fortuitum subsp. thermophilum," and stated that there are several biovars within the type subspecies. Recently, Grange and Stanford $(10,22)$ and Pattyn et al. (16) reported that there are several subtypes of M. fortuitum. Grange and Stanford (10) stated 
that, of seven serovars, serovar I was most frequently associated with disease and was associated with biovar $A$ of Bönicke, lipid type $F$, agglutination type $F$, and sensitin type $3(10,16)$. Thus, the second purpose of the present study was to determine whether there are some distinct subgroups, especially within the species $M$. fortuitum.

\section{MATERIALS AND METHODS}

Test strains. One hundred and sixty-five strains of rapidly growing, nonphotochromogenic mycobacteria were studied (Table 1).

Characters tested. One hundred and four characters (Table 2) were determined for each of the test strains. The methods of determining the characters were reported previously $(25,26,31,33)$.

Numerical classification. The matching coefficient ( $M$ value) between any two strains was calculated by the equation $M$ value $(\%)=\left(n_{s} \times 100 \%\right) /\left(n_{s}+n_{d}\right)$ where $n_{s}$ is the number of characters that show similar code symbols (++ or --) and $n_{d}$ is the number of characters which show different code symbols (+-). Clustering was done by the single-linkage method (20). The computer used was the IBM S-370, model 135, which is located in the Nagoya Factory of the Sumitomo Machinery Co., Obu, Aichi, Japan. The data for the original "strain $\mathrm{x}$ characters" matrix have been deposited with the World Data Center for Microorganisms, Brisbane, Australia.

Two methods of numerical classification were carried out, one using all 104 characters and another using 84 of the characters; omitted were 9 characters (no. 1 through 4, 9, and 64 through 67 ) ineffective in differentiating the strains; 4 characters (no. $30,31,35$, and 36) which showed positive matches in almost all (but not all) strains; 3 characters (no. 26, 34, and 46) related to others; and 4 characters (no. 8, 98, 100, 101) in which the interpretation of results was not clear-cut.

Association with disease. Thirteen of the strains of $M$. fortuitum were considered to be associated with disease. Of these, two were Cruz's classical strains that were isolated from cold abscesses of humans (ATCC 6841 and ATCC 6842), two were isolated from lymphadenitis of swine, and nine were isolated from sputum specimens from patients who were considered to have disease due to $M$. fortuitum. Within 3 months, the nine patients each showed at least three isolations, one of which was used for this study, and, concomitantly with these isolations, the appearance of cavitary lesions in their lungs; these isolates were collected through the Mycobacteriosis Research Group of the Japanese National Chest Hospitals.

\section{RESULTS}

Almost similar results were obtained in the classification of the study strains by means of two numerical taxonomy analyses, one involving 104 characters and the other involving 84 . The differentiation between strains received as $M$. fortuitum and those received as $M$. chelone $i$ was not so clear-cut in the former analysis. Hence, the results obtained in the latter analysis are shown in Fig. 1 to 3, wherein the differentiation between the two species is clear-cut.

Except for strains 104, 106, 107, and 108, the strains received as $M$. fortuitum, $M$. chelonei subsp. chelonei, or M. chelonei subsp. abscessus formed a cluster at a similarity level of $89 \%$. Strains received as $M$. smegmatis, $M$. chitae, or "M. agri" formed clusters at levels of 95,96 , and $92 \%$, respectively. Except for strains 125 and 132, the strains of $M$. smegmatis were divided into two subclusters at a level of $96 \%$. One contained strain ATCC 14468, which was suggested as the neotype strain for $M$. smegmatis in Bergey's Manual, 8th edition (17), and another contained strain ATCC 607.

Within the $\boldsymbol{M}$. fortuitum cluster, four small subclusters were observed, FOR1A, FOR1B, FOR2, and FOR3; about one-half of the strains did not form any subcluster. The first two subclusters belonged to the same subcluster according to the analysis in which 104 characters were used, but they could be differentiated in the analysis involving 84 characters.

The results obtained in this study show that the strains labeled " $M$. agri" form a distinct cluster, and these are here regarded as belonging to a distinct species. Because the name " $M$. agri" was not included on the Approved Lists (19), it is proposed here as a revived name for the same organism for which it was previously used $(30,32)$. A description of this organism is presented below.

Characters useful in distinguishing between the species of rapidly growing, nonphotochromogenic mycobacteria. As mentioned above, cluster formation was more clearcut in the taxonomic analysis in which 84 characters were used. Therefore, the characters of the clusters (species) obtained in this analysis are shown in Table 2. Table 3 shows distinguishing characters of the subclusters of $M$. fortuitum.

Relationship between the association with disease and the biovar in the $M$. fortuitum strains. Subclusters FOR1A, FOR1B, FOR2, and FOR3 of $M$. fortuitum contained 11, 9,4 , and 5 strains, respectively. The number of strains associated with disease contained in these subclusters were three, one, one, and two, respectively. Therefore, there was no significant difference among the percentages of the diseaseassociated strains ( $\chi^{2}$ test; $P>0.05$ ). Furthermore, correlations of disease with the amidase patterns, the patterns of utilization of nitrogen compounds as simultaneous nitrogen and carbon sources, and the patterns of utilization of carbohydrates as the carbon source in the presence of ammoniacal nitrogen were tested with these 
TABLE 1. Strains used

\begin{tabular}{|c|c|c|c|c|c|}
\hline \multirow{2}{*}{$\begin{array}{l}\text { Serial } \\
\text { no. }\end{array}$} & \multirow{2}{*}{$\begin{array}{l}\text { Labora- } \\
\text { tory no. }\end{array}$} & \multicolumn{2}{|c|}{ Received as: } & \multirow{2}{*}{ Source $^{a}$} & \multirow{2}{*}{ Habitat } \\
\hline & & Name & Strain & & \\
\hline 1 & E8363 & Mycobacterium fortuitium & & 1 & Sputum, humans \\
\hline 2 & E8366 & M. fortuitum & & 1 & Sputum, humans \\
\hline 3 & E8296 & M. fortuitum & & 1 & Sputum, humans \\
\hline 4 & E8359 & M. fortuitum & & 1 & Sputum, humans \\
\hline 5 & E8292 & $M$. fortuitum & & 1 & Sputum, humans \\
\hline 6 & E8361 & M. fortuitum & & 1 & Sputum, humans \\
\hline 7 & 18109 & M. fortuitum & ATCC 6842 & 2 & $\begin{array}{l}\text { Humans, cold } \\
\text { Abscess }^{b}\end{array}$ \\
\hline 8 & 18112 & M. fortuitum & ATCC 6841 (type) & 2 & $\begin{array}{l}\text { Humans, cold } \\
\text { Abscess }^{b}\end{array}$ \\
\hline 9 & 18224 & M. fortuitum & E7921 & 1 & Sputum, humans \\
\hline 10 & 42001 & "M. minetti" & ATCC 19542 (type) & 2 & Cow \\
\hline 11 & E9101 & M. fortuitum & Patient 1 & 1 & Sputum, humans ${ }^{b}$ \\
\hline 12 & 18077 & M. fortuitum & R48 & 3 & ? \\
\hline 13 & 18080 & M. fortuitum & $\mathrm{R} 449$ & 3 & ? \\
\hline 14 & 18083 & M. fortuitum & R520 & 3 & ? \\
\hline 15 & 18096 & M. fortuitum & R945 & 3 & $?$ \\
\hline 16 & 18001 & M. fortuitum & ATCC 19709 & 2 & Soil \\
\hline 17 & 18158 & M. forutitum & E5792 & 1 & Swine, lymph nodes ${ }^{b}$ \\
\hline 18 & 18005 & M. fortuitum & E302 & 1 & Soil \\
\hline 19 & 18010 & M. fortuitum & R389 & 3 & $?$ \\
\hline 20 & 18082 & M. fortuitum & R490 & 3 & ? \\
\hline 21 & 18002 & M. fortuitum & E334 & 1 & Soil \\
\hline 22 & 18004 & M. fortuitum & E313 & 1 & Soil \\
\hline 23 & 18054 & M. fortuitum & E1294 & 1 & Soil \\
\hline 24 & 18102 & M. fortuitum & R1028 & 3 & $?$ \\
\hline 25 & 18071 & M. fortuitum & NCTC 1542 & 3 & $?$ \\
\hline 26 & E8276 & M. fortuitum & & 1 & Sputum, humans \\
\hline 27 & E8864 & M. fortuitum & Patient 2 & 1 & Sputum, humans ${ }^{b}$ \\
\hline 28 & E9099 & M. fortuitum & & 1 & Sputum, humans \\
\hline 29 & E9100 & M. fortuitum & & 1 & Sputum, humans \\
\hline 30 & E8298 & M. fortuitum & & 1 & Sputum, humans \\
\hline 31 & 18212 & M. fortuitum & E7615 (Kamada) & 1 & Sputum, humans ${ }^{b}$ \\
\hline 32 & E8299 & M. fortuitum & & 1 & Sputum, humans \\
\hline 33 & 18231 & M. fortuitum & E8000 & 1 & Sputum, humans \\
\hline 34 & 18097 & M. fortuitum & R962 & 3 & $?$ \\
\hline 35 & 18221 & M. fortuitum & E7865 & 1 & Sputum, humans \\
\hline 36 & E6029 & M. fortuitum & & 1 & Sputum, humans \\
\hline 37 & E6023 & M. fortuitum & & 1 & Sputum, humans \\
\hline 38 & E6081 & M. fortuitum & Patient 3 & 1 & Sputum, humans ${ }^{b}$ \\
\hline 39 & 18003 & M. fortuitum & E330 & 1 & Soil \\
\hline 40 & 18209 & M. fortuitum & E7252 & 1 & Sputum, humans \\
\hline 41 & 18233 & M. fortuitum & E8112 & 1 & Sputum, humans \\
\hline 42 & 18085 & M. fortuitum & R640 & 3 & $?$ \\
\hline 43 & 18034 & M. fortuitum & E1274 & 1 & Soil \\
\hline 44 & 18035 & M. fortuitum & E1275 & 1 & Soil \\
\hline 45 & 18073 & "M. giae" & ATCC 11440 (type) & 2 & Anuran organ \\
\hline 46 & 18074 & "M. peregrinum" & ATCC 14467 (type) & 2 & Humans \\
\hline 47 & 18229 & M. fortuitum & E7976 (Matsui) & 1 & Sputum, humans ${ }^{b}$ \\
\hline 48 & 18007 & M. fortuitum & E308 & 1 & Soil \\
\hline 49 & 18089 & M. fortuitum & R681 & 3 & $?$ \\
\hline 50 & 18099 & M. fortuitum & R965 & 3 & ? \\
\hline 51 & 18105 & M. fortuitum & R1208 & 3 & $?$ \\
\hline 52 & 18223 & $M$. fortuitum & E7919 (Matsumoto) & 1 & Sputum, humans ${ }^{b}$ \\
\hline 53 & 18230 & M. fortuitum & E7994 & 1 & Sputum, humans \\
\hline 54 & E9082 & M. fortuitum & & 1 & Sputum, humans \\
\hline 55 & E2671 & M. fortuitum & & $\mathbf{l}$ & Sputum, humans \\
\hline 56 & 18009 & M. fortuitum & NCTC 8697 & 4 & ? \\
\hline 57 & 18087 & M. fortuitum & R645 & 3 & \\
\hline 58 & E8291 & M. fortuitum & & 1 & Sputum, humans \\
\hline 59 & 18090 & M. fortuitum & R720 & 1 & $?$ \\
\hline 60 & 18098 & M.fortuitum & R963 & 3 & $?$ \\
\hline 61 & 18180 & "M. fortuitum subsp. thermophilum" & ATCC 27408 & 2 & Soil \\
\hline 62 & 18205 & M. fortuitum & E7064 & 1 & Sputum, humans \\
\hline 63 & 18225 & M. fortuitum & E7922 & 1 & Sputum, humans \\
\hline 64 & E5963 & M. fortuitum & Patient 4 & 1 & Sputum, humans ${ }^{b}$ \\
\hline 65 & E5766 & $M$. fortuitum & Nishikido & 1 & Sputum, humans ${ }^{b}$ \\
\hline
\end{tabular}


TABLE 1.-Continued

\begin{tabular}{|c|c|c|c|c|c|}
\hline \multirow{2}{*}{$\begin{array}{c}\text { Serial } \\
\text { no. }\end{array}$} & \multirow{2}{*}{$\begin{array}{l}\text { Labora- } \\
\text { tory no. }\end{array}$} & \multicolumn{2}{|c|}{ Received as: } & \multirow{2}{*}{ Source $^{a}$} & \multirow{2}{*}{ Habitat } \\
\hline & & Name & Strain & & \\
\hline 66 & E8378 & M. fortuitum & & 1 & Sputum, humans \\
\hline 67 & 18207 & M. fortuitum & E7119 & 1 & Sputum, humans \\
\hline 68 & E8379 & M. fortuitum & & 1 & Sputum, humans \\
\hline 69 & E9020 & M. fortuitum & Hayashida & 1 & Sputum, humans ${ }^{b}$ \\
\hline 70 & E8323 & M. fortuitum & & 1 & Sputum, humans \\
\hline 71 & E8376 & M. fortuitum & & 1 & Sputum, humans \\
\hline 72 & E9061 & M. fortuitum & & 1 & Sputum, humans \\
\hline 73 & E8364 & M. fortuitum & & 1 & Sputum, humans \\
\hline 74 & 18103 & M. fortuitum & R1202 & 3 & ? \\
\hline 75 & 18220 & M. fortuitum & E7826 & 1 & Sputum, humans \\
\hline 76 & 18222 & M. fortuitum & E7916 & 1 & Sputum, humans \\
\hline 77 & 18226 & M. fortuitum & E7923 & 1 & Sputum, humans \\
\hline 78 & 44002 & M. fortuitum? & E5782 & 1 & Swine, lymph nodes ${ }^{b}$ \\
\hline 79 & 22001 & "M. runyonii" & $380 \mathrm{~s}$ & 4 & Humans \\
\hline 80 & 22011 & "M. runyonii" & ATCC 14472 (type) & 2 & Humans \\
\hline 81 & 22012 & "M. abscessus" & ATCC 19977 (type) & 2 & Human abscesses $^{b}$ \\
\hline 82 & 22014 & "M. abscessus" & ATCC 23007 & 2 & ? \\
\hline 83 & 22019 & "M. abscessus" & ATCC 23045 & 2 & $?$ \\
\hline 84 & 22021 & "M. abscessus" & E2846 & 1 & Sputum, humans \\
\hline 85 & 22003 & "M. runyonii" & $518 \mathrm{r}$ & 4 & Humans \\
\hline 86 & 22015 & "M. abscessus" & ATCC 23016 & 2 & $?$ \\
\hline 87 & 22023 & "M. abscessus" & E5359 & 1 & Sputum, humans \\
\hline 88 & 22002 & “M. runyonii?" & $481 r$ & 4 & Humans \\
\hline 89 & 22004 & "M. abscessus" & RJ-1 & 1 & Sputum, humans ${ }^{b}$ \\
\hline 90 & 19035 & M. chelone $i$ subsp. chelonei & E5870 & 1 & Sputum, humans \\
\hline 91 & 19040 & M. chelonei subsp. chelonei & ATCC 23000 & 2 & $?$ \\
\hline 92 & 19041 & M. chelonei & ATCC 23013 & 2 & ? \\
\hline 93 & 19036 & M. chelonei subsp. chelonei & E5880 & 1 & Sputum, humans \\
\hline 94 & 19044 & "M. borstelense" & ATCC 19235 (type) & 2 & Soil or water \\
\hline 95 & 19045 & "M. borstelense" & ATCC 19237 & 2 & Soil or water \\
\hline 96 & 19001 & "M. borstelense" & SN281 & 5 & Soil or water \\
\hline 97 & 19054 & M. chelonei & NCTC 946 (type) & 6 & Cold blood animal \\
\hline 98 & 22008 & "M. abscessus" & RJ-2 & 1 & Sputum, humans ${ }^{b}$ \\
\hline 99 & E7918 & M. chelonei subsp. chelonei & Patient 5 & 1 & Sputum, huamns ${ }^{b}$ \\
\hline 100 & 22031 & M. chelonei? & & 1 & Sputum, humans ${ }^{b}$ \\
\hline 101 & 19037 & M. chelonei & E6089 & 1 & Sputum, humans \\
\hline 102 & 22029 & "M. abscessus" & E7633 & 1 & Sputum, humans \\
\hline 103 & 18232 & M. fortuitum? & E8061 & 1 & Sputum, humans \\
\hline 104 & 19032 & M. chelonei & E5301 & 1 & Sputum, humans \\
\hline 105 & 19033 & M. chelonei & E5377 & 1 & Sputum, humans \\
\hline 106 & 19038 & M. chelonei subsp. chelonei & E6535 (Kato) & 1 & Resected lung \\
\hline 107 & 22028 & M. chelonei subsp. abscessus & E7556 & 1 & Lesions, humans \\
\hline 108 & 22030 & M. chelonei? & E7674 & 1 & Sputum, humans \\
\hline 109 & 41501 & "M. farcinogenes var. tchadense" & NCTC 10955 & 7 & Sputum, humans \\
\hline 110 & 18256 & "M. farcinogenes var. senegalense" & NCTC 10956 & 7 & ? \\
\hline 111 & 17005 & $M$. smegmatis & SN5 & 5 & ? \\
\hline 112 & 17014 & M. smegmatis & Wa237 & 8 & $?$ \\
\hline 113 & 17017 & M. smegmatis & R1103 & 8 & $?$ \\
\hline 114 & 17002 & M. smegmatis & SN2 & 5 & ? \\
\hline 115 & 17007 & M. smegmatis & SN7 & 5 & $?$ \\
\hline 116 & 17010 & M. smegmatis & SN10 & 5 & $?$ \\
\hline 117 & 17016 & M. smegmatis & R1102 & 8 & $?$ \\
\hline 118 & 17008 & M. smegmatis & SN8 & 5 & $?$ \\
\hline 119 & 17011 & M. smegmatis & Wa63 & 8 & $?$ \\
\hline 120 & 17012 & M. smegmatis & $\mathrm{Wa} 290$ & 8 & $?$ \\
\hline 121 & 17015 & M. smegmatis & Takeo & 1 & Chickens \\
\hline 122 & 17018 & M. smegmatis & $\mathrm{CDC}$ & 8 & $?$ \\
\hline 123 & 17022 & M. smegmatis & Denken & 1 & Chickens \\
\hline 124 & 17023 & M. smegmatis & Jucho & 1 & Chickens \\
\hline 125 & 17027 & $\begin{array}{l}\text { Mycobacterium sp. } \\
\text { M. smegmatis }\end{array}$ & $\begin{array}{l}\text { ATCC } 14468 \text { (suggested } \\
\text { neotype) }\end{array}$ & 2 & $?$ \\
\hline 126 & 17032 & M. smegmatis & ATCC 23019 & 2 & $?$ \\
\hline 127 & 45001 & M. smegmatis & ATCC 607 & 2 & ? \\
\hline 128 & 17035 & M. smegmatis & ATCC 23037 & 2 & $?$ \\
\hline 129 & 17036 & M. smegmatis & ATCC 23038 & 2 & ? \\
\hline 130 & 17034 & M. smegmatis & ATCC 23032 & 2 & $?$ \\
\hline 131 & 17031 & M. smegmatis & ATCC 23011 & 2 & ? \\
\hline
\end{tabular}


TABLE 1.-Continued

\begin{tabular}{|c|c|c|c|c|c|}
\hline \multirow{2}{*}{$\begin{array}{c}\text { Serial } \\
\text { no. }\end{array}$} & \multirow{2}{*}{$\begin{array}{l}\text { Labora- } \\
\text { tory no. }\end{array}$} & \multicolumn{2}{|c|}{ Received as: } & \multirow{2}{*}{ Source $^{a}$} & \multirow{2}{*}{ Habitat } \\
\hline & & Name & Strain & & \\
\hline 132 & 17033 & M. smegmatis & ATCC 23028 & 2 & $?$ \\
\hline 133 & 31003 & M. chitae & ATCC 19628 & 2 & Chicken organs \\
\hline 134 & 31004 & M. chitae & ATCC 19629 & 2 & Chicken organs \\
\hline 135 & 31005 & M. chitae & ATCC 19630 & 2 & Chicken organs \\
\hline 136 & 31002 & M. chitae & ATCC 19627 (type) & 2 & Chicken organs \\
\hline 137 & 90021 & "M. agri" & E6224 & 1 & Soil \\
\hline 138 & 90023 & "M. agri" & E6226 & 1 & Soil \\
\hline 139 & 90017 & "M. agri" & E6219 & 1 & Soil \\
\hline 140 & 90004 & "M. agri" & E6206 & 1 & Soil \\
\hline 141 & 90011 & "M. agri" & E6213 & 1 & Soil \\
\hline 142 & 90012 & "M. agri" & ATCC 27406 (type) & 1 & Soil \\
\hline 143 & 90013 & "M. agri" & E6215 & 2 & Soil \\
\hline 144 & 90015 & "M. agri" & E6217 & 1 & Soil \\
\hline 145 & 90016 & "M. agri" & E6218 & 1 & Soil \\
\hline 146 & 90009 & "M. agri" & E6221 & 1 & Soil \\
\hline 147 & 90010 & "M. agri" & E6212 & 1 & Soil \\
\hline 148 & 90008 & "M. agri" & E6210 & 1 & Soil \\
\hline 149 & 90003 & "M. agri" & E6205 & 1 & Soil \\
\hline 150 & 90019 & "M. agri" & E6222 & 1 & Soil \\
\hline 151 & 90024 & "M. agri" & E6227 & 1 & Soil \\
\hline 152 & 90025 & "M. agri" & E6228 & 1 & Soil \\
\hline 153 & 90006 & "M. agri" & E6208 & 1 & Soil \\
\hline 154 & 90014 & "M. agri" & E6216 & 1 & Soil \\
\hline 155 & 90026 & "M. agri" & E6230 & 1 & Soil \\
\hline 156 & 90001 & "M. agri" & E6203 & 1 & Soil \\
\hline 157 & 90005 & "M. agri" & E6207 & 1 & Soil \\
\hline 158 & 90031 & "M. agri" & E6238 & 1 & Soil \\
\hline 159 & 90028 & "M. agri" & E6232 & 1 & Soil \\
\hline 160 & 90029 & "M. agri" & E6233 & 1 & Soil \\
\hline 161 & 90007 & "M. agri" & E6209 & 1 & Soil \\
\hline 162 & 90018 & "M. agri" & ATCC 27407 & 2 & Soil \\
\hline 163 & 90027 & "M. agri" & E6231 & 1 & Soil \\
\hline 164 & 90030 & "M. agri" & E6234 & 1 & Soil \\
\hline 165 & 90002 & "M. agri" & E6204 & 1 & Soil \\
\hline
\end{tabular}

${ }^{a}$ 1, M. Tsukamura, National Chubu Hospital, Obu, Aichi, Japan; 2, American Type Culture Collection, Rockville, Md.; 3, R. E. Gordon, Rutgers University, New Burnswick, N.J.; 4, K. Takeya, Kyushu University, Fukuoka, Japan; 5, R. Bönicke, Forschungsinstitut Borstel, Borstel, West Germany; 6, J. L. Stanford, Middlesex Hospital Medical School, London, England; 7, M. P. Lechevalier, Rutgers University; 8, H. Saito, Shimaine Medical College, Izumo, Shimane, Japan.

${ }^{b}$ These are the strains considered to have caused infection in humans (or, in two cases, in swine).

strains. There was no correlation of disease with any of these characters (Table 4).

\section{DISCUSSION}

The results presented here show that the strains of " $M$. agri" tested form a distinct cluster when analyzed by numerical taxonomy methods, and thus " $M$. agri" is a valid species of rapidly growing, nonphotochromogenic mycobacteria. The same finding was shown previously but with only several strains (39). M. agri is therefore the seventh species of the rapidly growing, nonphotochromogenic mycobacteria after the following six species: $M$. smegmatis, $M$. fortuitum, $M$. chelonei, $M$. chitae, $M$. farcinogenes, and $M$. senegalense (19).

Two strains, 109 and 110, received as $M$. farcinogenes appeared to be distinct from each other. In 1979, Chamoiseau (6) divided this species into two species, $M$. farcinogenes and $M$. senegalense. Strains 109 and 110 are the type strains of the above-mentioned species, respectively (6). The results of the present study agreed with the finding of Chamoiseau. Both strains showed filamentous forms and, like the strains of $M$. chelone $i$ subsp. chelone $i$ and unlike the strains of the other species, were not tolerant to picric acid; strain 110 degraded $p$-aminosalicylate and formed a black formazan.

As mentioned above, the second method of analysis, in which 84 characters were used, resulted in a more clear-cut clustering. Therefore, we should like to discuss the results obtained by the second method.

M. fortuitum, M. chelonei subsp. chelonei, and $M$. chelonei subsp. abscessus formed clusters at the level of $89 \%$, and these were connected with each other and formed one cluster at a level of $88 \%$. These three taxa appeared to be closely related to each other and to be distinct from the other named taxa studied. Therefore, they may be regarded as a complex. In fact the strains of 
TABLE 2. Characters of rapidly growing, nonphotochromogenic mycobacteria ${ }^{a}$

\begin{tabular}{|c|c|c|c|c|c|c|c|}
\hline \multirow[b]{2}{*}{ Character } & \multicolumn{7}{|c|}{$\%$ of strains showing positive reaction } \\
\hline & $\begin{array}{c}\text { M. fortui- } \\
\text { tum } \\
(n=78)\end{array}$ & $\begin{array}{c}M \text {. chelonei } \\
\text { subsp. } a b- \\
\text { scessus } \\
(n=14)\end{array}$ & $\begin{array}{c}\text { M. chelonei } \\
\text { subsp. che- } \\
\text { lonei } \\
(n=12)\end{array}$ & $\begin{array}{c}\text { M. smeg- } \\
\text { matis } \\
\text { subsp. } \\
\text { smegmatis } \\
(n=15)\end{array}$ & $\begin{array}{c}M . \text { smeg- } \\
\text { matis } \\
\text { subsp. lac- } \\
\text { ticola } \\
(n=5)\end{array}$ & $\begin{array}{l}\text { M. chitae } \\
(n=4)\end{array}$ & $\begin{array}{l}\text { M. agri } \\
(n=29)\end{array}$ \\
\hline 1. Strong acid-fastness & 95 & 100 & 92 & 100 & 100 & 100 & 100 \\
\hline 2. Weak or partial acid-fastness & 100 & 100 & 100 & 100 & 100 & 100 & 100 \\
\hline 3. Permanent mycelium & 0 & 0 & 0 & 0 & 0 & 0 & 0 \\
\hline 4. Fragmenting mycelium & 0 & 0 & 0 & 0 & 0 & 0 & 0 \\
\hline $\begin{array}{l}\text { 5. Long rods (more than } 7 \mu \mathrm{m} \text { in } \\
\text { length) }\end{array}$ & 8 & 0 & 0 & 80 & 100 & 0 & 86 \\
\hline $\begin{array}{l}\text { 6. Intermediate rods ( } 3-6 \mu \mathrm{m} \text { in } \\
\text { length) }\end{array}$ & 87 & 100 & 83 & 100 & 100 & 0 & 100 \\
\hline $\begin{array}{l}\text { 7. Short rods (less than } 2 \mu \mathrm{m} \text { in } \\
\text { length) }\end{array}$ & 94 & 100 & 100 & 100 & 100 & 0 & 45 \\
\hline 8. Cross-barring & 0 & 0 & 0 & 0 & 0 & 0 & 0 \\
\hline 9. Cord formation & 0 & 0 & 0 & 0 & 0 & 0 & 0 \\
\hline 10. Rough colonies & 71 & 64 & 42 & 27 & 100 & 0 & 97 \\
\hline 11. Colony pigmentation in dark & 0 & 0 & 0 & 0 & 0 & 0 & 0 \\
\hline 12. Photochromogenicity & 0 & 0 & 0 & 0 & 0 & 0 & 0 \\
\hline $\begin{array}{l}\text { 13. Growth at } 3 \text { days (rapid } \\
\text { growth) }\end{array}$ & 100 & 100 & 100 & 100 & 100 & 100 & 100 \\
\hline 14. Growth at $28^{\circ} \mathrm{C}$ & 100 & 100 & 100 & 100 & 100 & 100 & 100 \\
\hline 15. Growth at $37^{\circ} \mathrm{C}$ & 100 & 100 & 100 & 100 & 100 & 100 & 100 \\
\hline 16. Growth at $45^{\circ} \mathrm{C}$ & 9 & 0 & 0 & 100 & 100 & 0 & 100 \\
\hline 17. Growth at $52^{\circ} \mathrm{C}$ & 0 & 0 & 0 & 0 & 60 & 0 & 7 \\
\hline $\begin{array}{l}\text { 18. Resistance to } 0.2 \% p \text {-amino- } \\
\text { salicylate }\end{array}$ & 100 & 100 & 100 & 100 & 100 & 100 & 100 \\
\hline $\begin{array}{l}\text { 19. Degradation of } p \text {-aminosalicy- } \\
\text { late }\end{array}$ & 65 & 100 & 100 & 0 & 0 & 0 & 0 \\
\hline $\begin{array}{l}\text { 20. Resistance to } \mathrm{NH}_{2} \mathrm{OH} \cdot \mathrm{HCl} \\
(125 \mu \mathrm{g} / \mathrm{ml})\end{array}$ & 100 & 100 & 100 & 100 & 100 & 100 & 69 \\
\hline $\begin{array}{l}\text { 21. Resistance to } \mathrm{NH}_{2} \mathrm{OH} \cdot \mathrm{HCl} \\
(250 \mu \mathrm{g} / \mathrm{ml})\end{array}$ & 100 & 100 & 100 & 60 & 100 & 100 & 0 \\
\hline $\begin{array}{l}\text { 22. Resistance to } \mathrm{NH}_{2} \mathrm{OH} \cdot \mathrm{HCl} \\
(500 \mu \mathrm{g} / \mathrm{ml})\end{array}$ & 100 & 100 & 100 & 0 & 0 & 0 & 0 \\
\hline 23. Growth on Sauton agar & 100 & 100 & 100 & 100 & 100 & 100 & 100 \\
\hline $\begin{array}{l}\text { 24. Tolerance to } 0.1 \% \text { sodium sali- } \\
\text { cylate (Sauton agar) }\end{array}$ & 100 & 100 & 25 & 100 & 100 & 0 & 7 \\
\hline 25. Degradation of salicylate & 67 & 93 & 58 & 0 & 0 & 0 & 0 \\
\hline $\begin{array}{l}\text { 26. Tolerance to } 0.1 \% \text { picric acid } \\
\text { (Sauton agar) }\end{array}$ & 100 & 93 & 25 & 100 & 100 & 100 & 100 \\
\hline $\begin{array}{l}\text { 27. Tolerance to } 0.2 \% \text { picric acid } \\
\text { (Sauton agar) }\end{array}$ & 99 & 93 & 8 & 100 & 100 & 100 & 100 \\
\hline 28. Arylsulfatase (3 days) & 94 & 93 & 100 & 87 & 20 & 0 & 24 \\
\hline 29. Arylsulfatase (2 weeks) & 100 & 100 & 100 & 100 & 100 & 100 & 100 \\
\hline $\begin{array}{l}\text { 30. Tolerance to thiophene-2-car- } \\
\text { boxylic acid hydrazide (1 } \\
\mu \mathrm{g} / \mathrm{ml})\end{array}$ & 100 & 100 & 100 & 100 & 100 & 100 & 100 \\
\hline 31. Resistance to $0.05 \%$ salicylate & 100 & 100 & 100 & 100 & 100 & 100 & 100 \\
\hline $\begin{array}{l}\text { 32. Resistance to ethambutol (5 } \\
\mu \mathrm{g} / \mathrm{ml})\end{array}$ & 99 & 100 & 100 & 93 & 100 & 100 & 0 \\
\hline $\begin{array}{l}\text { 33. Tolerance to } 0.1 \% \mathrm{NaNO}_{2} \\
\text { (Sauton agar) }\end{array}$ & 100 & 100 & 92 & 100 & 100 & 100 & 100 \\
\hline $\begin{array}{l}\text { 34. Tolerance to } 0.2 \% \mathrm{NaNO}_{2} \\
\text { (Sauton agar) }\end{array}$ & 100 & 100 & 83 & 100 & 100 & 100 & 100 \\
\hline $\begin{array}{l}\text { 35. Growth on } 1 \% \text { Tween-Sauton } \\
\text { agar }\end{array}$ & 100 & 100 & 92 & 100 & 100 & 100 & 100 \\
\hline $\begin{array}{l}\text { 36. Resistance to } p \text {-nitrobenzoic } \\
\text { acid }(0.5 \mathrm{mg} / \mathrm{ml})\end{array}$ & 100 & 100 & 100 & 100 & 100 & 100 & 100 \\
\hline $\begin{array}{l}\text { 37. Resistance to rifampin }(25 \mu \mathrm{g} / \\
\mathrm{ml})\end{array}$ & 100 & 100 & 100 & 100 & 100 & 100 & 100 \\
\hline 38. Niacin production & 0 & 0 & 8 & 0 & 0 & 0 & 0 \\
\hline 39. Tween hydrolysis (7 days) & 1 & 0 & 0 & 100 & 100 & 100 & 0 \\
\hline 40. Tween hydrolysis (14 days) & 8 & 7 & 0 & 100 & 100 & 100 & 100 \\
\hline 41. Catalase (semiquantitative) & 37 & 79 & 58 & 7 & 0 & 100 & 100 \\
\hline 42. $\alpha$-Esterase & 29 & 21 & 58 & 7 & 0 & 100 & 100 \\
\hline 43. $\beta$-Esterase & 63 & 100 & 100 & 7 & 0 & 100 & 100 \\
\hline 44. $\beta$-Galactosidase & 17 & 86 & 100 & 100 & 100 & 0 & 72 \\
\hline
\end{tabular}


TABLE 2.-Continued

\begin{tabular}{|c|c|c|c|c|c|c|c|}
\hline \multirow[b]{2}{*}{ Character } & \multicolumn{7}{|c|}{$\%$ of strains showing positive reaction } \\
\hline & $\begin{array}{c}\text { M. fortui- } \\
\text { tum } \\
(n=78)\end{array}$ & $\begin{array}{c}M \text {. chelonei } \\
\text { subsp. ab- } \\
\text { scessus } \\
(n=14)\end{array}$ & $\begin{array}{c}\text { M. chelonei } \\
\text { subsp. che- } \\
\text { lonei } \\
(n=12)\end{array}$ & $\begin{array}{l}M . \text { smeg- } \\
\text { matis } \\
\text { subsp. } \\
\text { smegmatis } \\
(n=15)\end{array}$ & $\begin{array}{l}\text { M. smeg- } \\
\text { matis } \\
\text { subsp. lac- } \\
\text { ticola } \\
(n=5)\end{array}$ & $\begin{array}{l}\text { M. chitae } \\
(n=4)\end{array}$ & $\begin{array}{l}\text { M. agri } \\
(n=29)\end{array}$ \\
\hline 45. Acid phosphatase & 35 & 7 & 33 & 0 & 0 & 100 & 3 \\
\hline 46. Nitrate reduction $(6 \mathrm{~h})$ & 95 & 7 & 0 & 100 & 100 & 100 & 93 \\
\hline 47. Nitrate reduction ( $24 \mathrm{~h}$ ) & 100 & 14 & 0 & 100 & 100 & 100 & 100 \\
\hline 48. Acetamidase & 87 & 14 & 8 & 93 & 0 & 100 & 66 \\
\hline 49. Benzamidase & 1 & 0 & 8 & 93 & 0 & 0 & 0 \\
\hline 50. Urease & 100 & 100 & 83 & 100 & 100 & 100 & 100 \\
\hline 51. Isonicotinamidase & 0 & 0 & 0 & 93 & 0 & 0 & 0 \\
\hline 52. Nicotinamidase & 64 & 50 & 50 & 93 & 0 & 100 & 100 \\
\hline 53. Pyrazinamidase & 67 & 57 & 33 & 100 & 0 & 100 & 100 \\
\hline 54. Salicylamidase & 0 & 0 & 0 & 13 & 0 & 0 & 0 \\
\hline 55. Allantoinase & 99 & 0 & 0 & 13 & 100 & 0 & 100 \\
\hline 56. Succinamidase & 0 & 0 & 0 & 93 & 20 & 0 & 0 \\
\hline 57. Glutamate as $\mathrm{N}$ and $\mathrm{C}$ sources & 100 & 100 & 100 & 100 & 100 & 100 & 100 \\
\hline 58. L-Serine as $N$ and $C$ sources & 56 & 100 & 75 & 100 & 100 & 0 & 0 \\
\hline $\begin{array}{l}\text { 59. Glucosamine as } \mathrm{N} \text { and } \mathrm{C} \\
\text { sources }\end{array}$ & 99 & 29 & 25 & 100 & 100 & 0 & 100 \\
\hline 60. Acetamide as $\mathrm{N}$ and $\mathrm{C}$ sources & 56 & 7 & 0 & 100 & 100 & 100 & 0 \\
\hline $\begin{array}{l}\text { 61. Benzamide as } \mathrm{N} \text { and } \mathrm{C} \\
\text { sources }\end{array}$ & 0 & 0 & 0 & 100 & 100 & 0 & 0 \\
\hline $\begin{array}{l}\text { 62. Monoethanolamine as } \mathrm{N} \text { and } \\
\mathrm{C} \text { sources }\end{array}$ & 99 & 100 & 100 & 100 & 100 & 0 & 0 \\
\hline $\begin{array}{l}\text { 63. Trimethylene diamine as } \mathrm{N} \\
\text { and } \mathrm{C} \text { sources }\end{array}$ & 100 & 86 & 0 & 100 & 100 & 0 & 0 \\
\hline $\begin{array}{l}\text { 64. Glucose as } \mathrm{C} \text { source (gluta- } \\
\text { mate- } N \text { ) }\end{array}$ & 100 & 100 & 100 & 100 & 100 & 100 & 100 \\
\hline $\begin{array}{l}\text { 65. Acetate as } \mathrm{C} \text { source (gluta- } \\
\text { mate-N) }\end{array}$ & 100 & 100 & 100 & 100 & 100 & 100 & 100 \\
\hline $\begin{array}{l}\text { 66. Succinate as } \mathrm{C} \text { source (gluta- } \\
\text { mate- } N \text { ) }\end{array}$ & 100 & 100 & 100 & 100 & 100 & 100 & 100 \\
\hline $\begin{array}{l}\text { 67. Pyruvate as C source (gluta- } \\
\text { mate-N) }\end{array}$ & 100 & 100 & 100 & 100 & 100 & 100 & 100 \\
\hline 68. Acetate as $\mathrm{C}$ source & 100 & 100 & 100 & 100 & 100 & 100 & 100 \\
\hline 69. Citrate as $\mathrm{C}$ source & 18 & 7 & 100 & 100 & 100 & 0 & 24 \\
\hline 70. Succinate as $\mathrm{C}$ source & 100 & 86 & 42 & 100 & 100 & 75 & 100 \\
\hline 71. Malate as $\mathrm{C}$ source & 100 & 86 & 33 & 100 & 100 & 50 & 100 \\
\hline 72. Pyruvate as $\mathrm{C}$ source & 100 & 100 & 83 & 100 & 100 & 100 & 100 \\
\hline 73. Benzoate as $\mathrm{C}$ source & 3 & 0 & 0 & 100 & 100 & 0 & 0 \\
\hline 74. Malonate as $\mathrm{C}$ source & 4 & 0 & 0 & 100 & 100 & 0 & 21 \\
\hline 75. Fumarate as $\mathrm{C}$ source & 100 & 57 & 25 & 100 & 100 & 0 & 100 \\
\hline 76. Fructose as $\mathrm{C}$ source & 100 & 71 & 58 & 100 & 100 & 75 & 100 \\
\hline 77. Sucrose as $\mathrm{C}$ source & 40 & 7 & 0 & 100 & 40 & 25 & 0 \\
\hline 78. Ethanol as $\mathrm{C}$ source & 91 & 0 & 0 & 100 & 100 & 0 & 69 \\
\hline 79. $n$-Propanol as C source & 100 & 14 & 0 & 100 & 100 & 50 & 93 \\
\hline 80. Propylene glycol as C source & 78 & 0 & 0 & 100 & 100 & 0 & 21 \\
\hline $\begin{array}{l}\text { 81. 1,3-Butylene glycol was } \mathrm{C} \\
\text { source }\end{array}$ & 71 & 0 & 0 & 100 & 100 & 0 & 0 \\
\hline $\begin{array}{l}\text { 82. 1,4-Butylene glycol was } \mathrm{C} \\
\text { source }\end{array}$ & 14 & 0 & 0 & 40 & 60 & 0 & 0 \\
\hline $\begin{array}{l}\text { 83. 2,3-Butylene glycol was C } \\
\text { source }\end{array}$ & 60 & 0 & 0 & 100 & 100 & 0 & 0 \\
\hline 84. $n$-Butanol as $\mathrm{C}$ source & 100 & 79 & 0 & 100 & 100 & 100 & 0 \\
\hline 85. Isobutanol as $\mathrm{C}$ source & 100 & 29 & 0 & 100 & 100 & 100 & 0 \\
\hline 86. Acid from glucose & 100 & 93 & 100 & 100 & 100 & 0 & 100 \\
\hline 87. Acid from mannose & 100 & 93 & 100 & 100 & 100 & 0 & 100 \\
\hline 88. Glucose as $\mathrm{C}$ source & 100 & 100 & 100 & 100 & 100 & 100 & 100 \\
\hline 89. Mannose as $C$ source & 100 & 100 & 100 & 100 & 100 & 0 & 100 \\
\hline 90. Galactose as C source & 0 & 0 & 0 & 100 & 100 & 0 & 0 \\
\hline 91. Arabinose as $\mathrm{C}$ source & 0 & 0 & 0 & 100 & 100 & 0 & 0 \\
\hline 92. Xylose as C source & 0 & 0 & 0 & 100 & 100 & 0 & 100 \\
\hline 93. Rhamnose as $\mathrm{C}$ source & 0 & 0 & 0 & 100 & 100 & 0 & 0 \\
\hline 94. Trehalose as $\mathrm{C}$ source & 41 & 36 & 50 & 100 & 100 & 0 & 0 \\
\hline 95. Inositol as $\mathrm{C}$ source & 13 & 0 & 0 & 100 & 100 & 0 & 100 \\
\hline 96. Mannitol as $\mathrm{C}$ source & 53 & 7 & 0 & 100 & 100 & 0 & 0 \\
\hline 97. Sorbitol as C source & 0 & 0 & 0 & 100 & 100 & 0 & 0 \\
\hline
\end{tabular}


TABLE 2.-Continued

\begin{tabular}{lccccccc}
\hline \multicolumn{1}{c}{ Character } & $\begin{array}{c}\text { M. fortui- } \\
\text { tum } \\
(n=78)\end{array}$ & $\begin{array}{c}\text { M. chelonei } \\
\text { subsp. } a b- \\
\text { scessus } \\
(n=14)\end{array}$ & $\begin{array}{c}\text { M. chelonei } \\
\text { subsp. che } \\
\text { lonei } \\
(n=12)\end{array}$ & $\begin{array}{c}\text { M. smeg- } \\
\text { matis } \\
\text { subsp. } \\
\text { smegmatis } \\
(n=15)\end{array}$ & $\begin{array}{c}\text { M. smeg- } \\
\text { matis } \\
\text { subsp. lac- } \\
\text { ticola } \\
(n=5)\end{array}$ & $\begin{array}{c}\text { M. chitae } \\
(n=4)\end{array}$ & $\begin{array}{c}M \text {. agri } \\
(n=29)\end{array}$ \\
\hline 98. Acetamide as N source & 90 & 100 & 50 & 100 & 100 & 100 & 86 \\
99. Benzamide as N source & 5 & 0 & 0 & 100 & 100 & 0 & 0 \\
100. Urea as N source & 95 & 100 & 42 & 100 & 100 & 100 & 69 \\
101. Pyrazinamide as N source & 91 & 100 & 33 & 100 & 100 & 100 & 100 \\
102. Nicotinamide as N source & 91 & 93 & 25 & 100 & 100 & 100 & 10 \\
103. Nitrate as N source & 92 & 100 & 33 & 100 & 100 & 100 & 3 \\
104. Nitrite as N source & 73 & 93 & 0 & 100 & 100 & 0 & 0
\end{tabular}

${ }^{a}$ Unless otherwise noted, resistance to any agent was tested in Ogawa egg medium. Unless otherwise noted, utilization of carbohydrates as carbon source was tested in the presence of ammoniacal nitrogen.

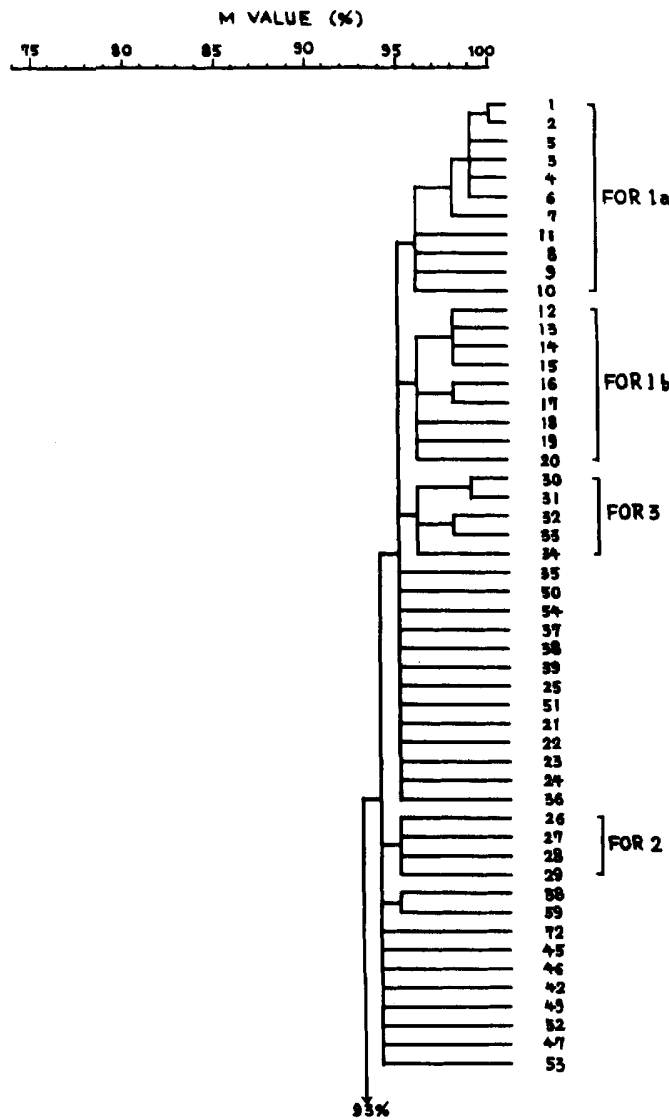

Fig. 1. Dendrogram prepared from results of $\mathrm{nu}$ merical classification of rapidly growing, nonphotochromogenic mycobacteria, using 84 characters. FOR1a, FOR1b, FOR3, and FOR2 are subclusters observed within the cluster of $M$. fortuitum strains.

these species had previously been considered as belonging to one species, $M$. fortuitum, by Gordon and Smith (9), and $M$. chelonei subsp. $a b$ scessus had been regarded as a subspecies of $M$.

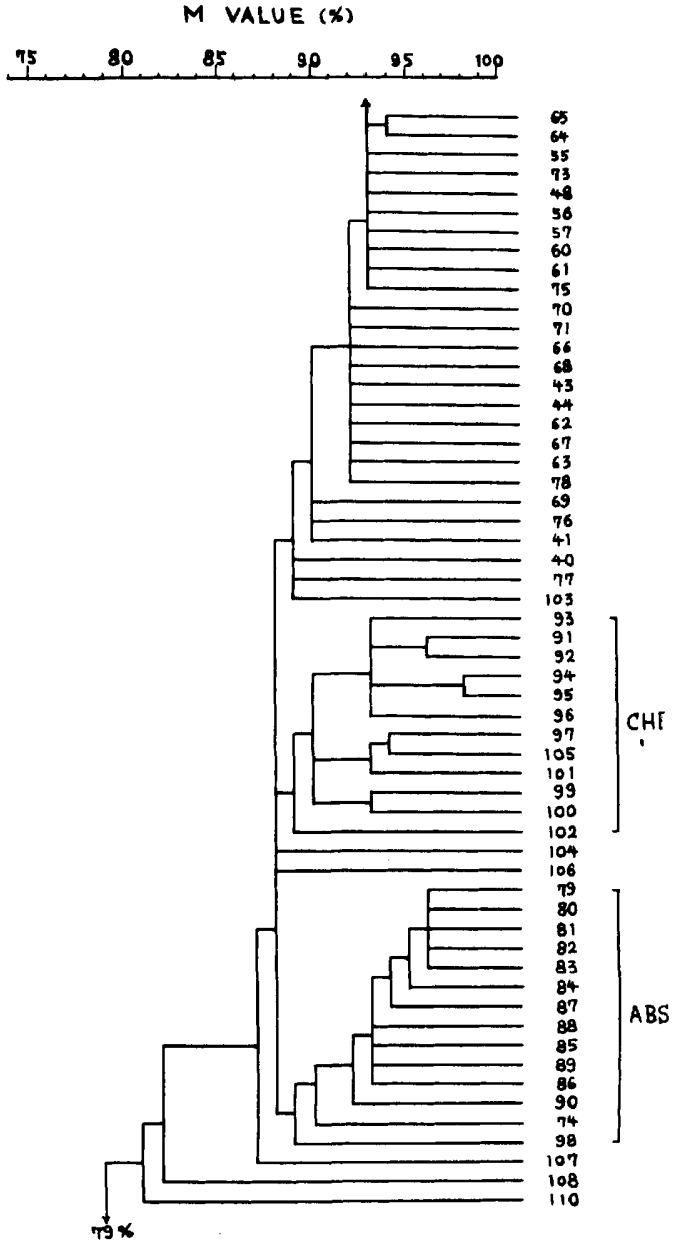

Fig. 2. Dendrogram prepared from results of $\mathrm{nu}$ merical classification of rapidly growing, nonphotochromogenic mycobacteria, using 84 characters. CHE and $A B S$ are clusters of $M$. chelonei subsp. chelonei and $M$. chelonei subsp. abscessus strains, respectively. 


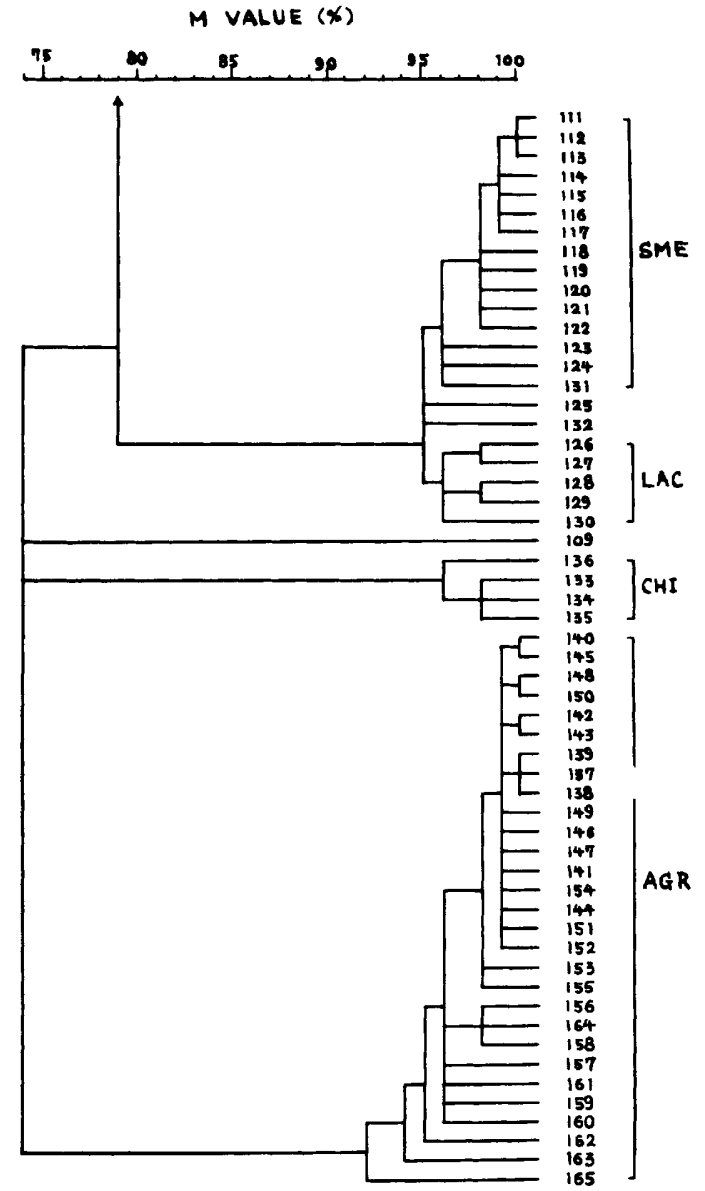

Fig. 3. Dendrogram prepared from results of numerical classification of rapidly growing, nonphotochromogenic mycobacteria, using 84 characters. $S M E, L A C, C H I$, and AGR are clusters of $M$. smegmatis subsp. smegmatis, "M. smegmatis subsp. lacticola," M. chitae, and "M. agri" strains, respectively. fortuitum by Tsukamura et al. (40). Bojalil et al. (3) differentiated between $M$. fortuitum and " $M$. runyonii" (M. chelonei subsp. abscessus), and Takeya et al. (24) differentiated between these two taxa by the skin reaction to tuberculins. Later, Tsukamura et al. (38) stated that " $M$. runyonii" and " $M$. abscessus" were the same organism and, according to the rule of priority, " $M$. runyonii" was a later synonym of " $M$. $a b$ scessus" Moore and Frerichs (15). Bönicke and Stottmeier (5) proposed a new species, "Mycobacterium borstelense," in 1965. In 1970, Tsukamura (28) regarded $M$. fortuitum, " $M$. absces . sus," and " $M$. borstelense" as distinct taxa on the basis of a numerical analysis of phenotypic characters. On the other hand, Stanford and Beck (21) stated that "M. abscessus," "M. borstelense," and the type strain of $M$. chelonei, which was maintained in the National Collection of Type Cultures, London, could not be differentiated by immunotaxonomic analysis and should be reduced to one taxon, $M$. chelonei. Stanford et al. (23) confirmed this finding. In contrast, Weiszfeiler et al. (42) observed a difference in virulence to mice between " $M$. abscessus" and "M. borstelense," which are now regarded as $M$. chelonei subsp. abscessus and $M$. chelonei subsp. chelonei, respectively. On the other hand, Jenkins et al. (12) could not differentiate between these two taxa on the basis of lipid analysis and seroagglutination reactions. Finally, an international cooperative study by Kubica et al. (13) revealed that these two taxa could be differentiated by a phenetic analysis but not by lipid and immunological analyses. Thus, they proposed that the taxa be two subspecies, $M$. chelonei subsp. chelonei and $M$. chelonei subsp. abscessus. In the phenetic analysis of the present study, the taxa $M$. fortuitum, $M$. chelonei subsp. chelonei, and $M$. chelonei subsp.

TABLE 3. Distinguishing characters among subgroups of $M$. fortuitum

\begin{tabular}{|c|c|c|c|c|}
\hline \multirow{2}{*}{ Character } & \multicolumn{4}{|c|}{$\%$ of strains showing positive reaction } \\
\hline & $\begin{array}{c}\text { FOR1a } \\
(n=11)\end{array}$ & $\begin{array}{l}\text { FOR1b } \\
(n=9)\end{array}$ & $\begin{array}{c}\text { FOR2 } \\
(n=4) \\
\end{array}$ & $\begin{array}{c}\text { FOR3 } \\
(n=5)\end{array}$ \\
\hline Degradation of $p$-aminosalicylate & 0 & 100 & 50 & 100 \\
\hline Degradation of salicylate & 0 & 100 & 25 & 100 \\
\hline Catalase (semiquantitative) & 45 & 0 & 75 & 100 \\
\hline$\beta$-Galactosidase & 0 & 0 & 100 & 0 \\
\hline Nicotinamidase & 73 & 0 & 100 & 100 \\
\hline Pyrazinamidase & 73 & 0 & 100 & 100 \\
\hline Serine as $\mathrm{N}$ and $\mathrm{C}$ sources & 100 & 22 & 0 & 80 \\
\hline Acetamide as $\mathbf{N}$ and $\mathbf{C}$ sources & 100 & 44 & 100 & 0 \\
\hline 1,3-Butylene glycol as $\mathrm{C}$ source & 91 & 67 & 75 & 0 \\
\hline Trehalose as C source & 9 & 89 & 0 & 40 \\
\hline Mannitol as $\mathrm{C}$ source & 0 & 44 & 0 & 100 \\
\hline No. of strains associated with disease & $3(27)^{a}$ & $1(11)$ & $1(25)$ & I (20) \\
\hline
\end{tabular}

\footnotetext{
a Percentage of strains associated with disease in each group.
} 
TABLE 4. Amidase patterns, patterns of utilization of nitrogen compounds as simultaneous nitrogen and carbon sources, and patterns of utilization of 10 carbohydrates as carbon sources of the 78 test strains of $M$. fortuitum, and their disease association

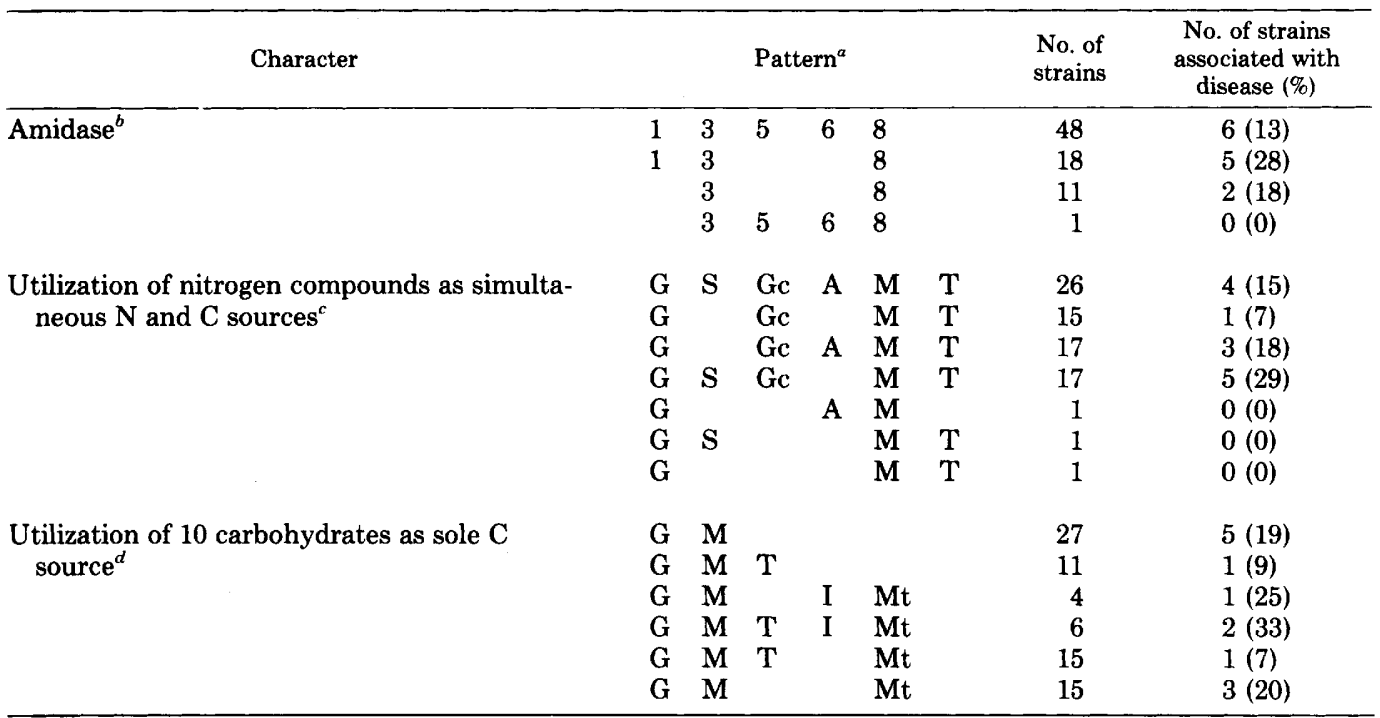

${ }^{a}$ Shown by a set of characters which have shown positive reaction. Characters in the footnotes which do not appear in the table showed a negative reaction.

${ }^{b} 1$, Acetamidase; 2 , benzamidase; 3 , urease; 4, isonicotinamidase; 5 , nicotinamidase; 6 , pyrazinamidase; 7 , salicylamidase; 8 , allantoinase; 9 , succinamidase.

${ }^{c} \mathrm{G}$, Glutamate; S, serine; Gc, glucosamine; A, acetamide; B, benzamide; M, monoethanolamine; $T$, trimethylenediamine.

${ }^{d} \mathrm{G}$, Glucose; M, mannose; Ga, galactose; A, arabinose; X, xylose; R, rhamnose; T, trehalose; I, inositol; Mt, mannitol; S, sorbitol.

abscessus appeared to be different taxa of the same rank, and the data suggest that they should be regarded as different species.

Grange and Stanford $(10,22)$ and Pattyn et al. (16) suggested that $M$. fortuitum be divided into several subgroups; one of the subgroups, which belonged to serovar I and was considered to be identical to biovar (biotype) A of Bönicke (4), was frequently associated with disease. Biovar A strains produce acid from glucose, mannose, and trehalose. In contrast, Haas et al. (11) stated that $\boldsymbol{M}$. fortuitum strains formed a homogeneous group when viewed from the standpoint of cell proteins, and Vanden Berghe and Pattyn (41) showed that $M$. fortuitum strains were a homogeneous group with respect to the electrophoretic patterns of their proteins. Binko and Bartholomew (2) observed that strains of $M$. fortuitum produced isozymes that were immunologically identical. The results of the present study show that several subgroups are present within the species $M$. fortuitum, but that the subgroups can accommodate only onehalf of the test strains. It was shown that each subgroup (Table 3) contained a few strains associated with disease and that none of the subgroups was statistically more significantly asso- ciated with disease than any other. We could also differentiate a number of biovars (Table 4), but none of these appeared to be significantly more associated with disease than any other.

M. smegmatis was considered to be a homogeneous taxon by Gordon and Smith (8). Tsukamura et al. $(29,37,38)$ showed the presence of two subgroups in this species, " $M$. smegmatis subsp. smegmatis" and " $M$. smegmatis subsp. lacticola." These taxa differ in their amidase patterns. The existence of the subgroups was confirmed in the present study.

Characters useful in differentiating the species included in the present study are shown in Table 2. Of these colony morphology is used frequently as a character helpful in differentiating between species. However, only the presence or absence of rough colonies in fresh isolates should be used as a distinguishing character, for, as shown previously with $M$. smegmatis (34), strains maintained in laboratories tend to show rough colonies, whereas freshly isolated strains usually produce smooth colonies.

M. agri (Tsukamura 1972) Tsukamura sp. nov., nom. rev. (ag'ri. Gr. n. agrus field; M. L. gen. $n$. agri of a field). The original isolate of this species was obtained from alkali-treated soil 
inoculated on egg media incubated at $42^{\circ} \mathrm{C}$. As shown in the present study, this taxon can readily be differentiated from all previously described species of rapidly growing, nonphotochromogenic mycobacteria. Furthermore, it is clearly different from all other mycobacteria, including the rapidly growing, scotochromogenic mycobacteria (39). Consequently, the name is herein revived for the same organism with which it was originally associated (30). The characters of this species are listed in Table 2.

The characters of the type strain 90012 (= ATCC 27406) are as follows. Acid-fast rods which often grow into long threads. Rough, nonpigmented colonies are produced in the dark and after exposure to light. Growth occurs after 3 days on egg media and at 28,37 , and $45^{\circ} \mathrm{C}$; there is no growth at $52^{\circ} \mathrm{C}$. Resistant to each of the following in egg media (Ogawa egg medium or Löwenstein-Jensen medium): $5 \% \mathrm{NaCl}, p$-nitrobenzoic acid $(0.5 \mathrm{mg} / \mathrm{ml})$; thiophene-2-carboxylic acid hydrazide $(1 \mu \mathrm{g} / \mathrm{ml})$. Niacin is not produced. Catalase positive (semiquantitative method). Tween not hydrolyzed after 10 days (slightly hydrolyzed after 14 days). $\beta$-Galactosidase is usually produced. Nitrate is reduced to nitrite. Susceptible to $\mathrm{NH}_{2} \mathrm{OH} \cdot \mathrm{HCl}(0.25 \mathrm{mg} /$ $\mathrm{ml}$ ) in egg media; tolerant to $0.2 \%$ picric acid in Sauton agar; tolerant to $0.1 \%$ sodium nitrite in Sauton agar. Arylsulfatase negative after 3 days but positive after 14 days. Produces $\alpha$ - and $\beta$ esterases but not acid phosphatase.

Amidase pattern: acetamidase, urease, nicotinamidase, pyrazinamidase, and allantoinase are produced; benzamidase, isonicotinamidase, salicylamidase, and succinamidase are not produced. Glutamate and glucosamine are utilized as simultaneous nitrogen and carbon sources; serine, acetamide, benzamide, monoethanolamine, and trimethylene diamine are not so utilized. Glucose, mannose, xylose, inositol, fructose, acetate, succinate, malate, pyruvate, fumarate, ethanol, $n$-propanol, and propylene glycol are utilized as carbon sources in the presence of ammoniacal nitrogen; galactose, arabinose, rhamnose, trehalose, mannitol, sorbitol, sucrose, citrate, benzoate, malonate, $n$-butanol, isobutanol, 1,3-, 1,4-, and 2,3-butylene glycols are not thus utilized. Nitrite is not utilized as a sole source of nitrogen in the presence of glycerol as a carbon source.

Source: Soil.

Type strain: 90012; a culture of this strain has been deposited in the American Type Culture Collection, Rockville, Md., as ATCC 27406.

\section{REPRINT REQUESTS}

Address reprint requests to: Dr. M. Tsukamura, The National Chubu Hospital, Obu, Aichi, Japan 474.

\section{LITERATURE CITED}

1. Bergey, D. H., F. C. Harrison, R. S. Breed, B. W. Hammer, and F. M. Hantoon. 1923. Bergey's manual of determinative bacteriology, 1st ed., p. 376. The Williams \& Wilkins Co., Baltimore.

2. Binko, B. P., and W. R. Bartholomew. 1978. Enzymatic and immunological characterization of the Mycobacte rium fortuitum complex. J. Clin. Microbiol. 8:246-251.

3. Bojalil, L. F., J. Cerbón, and A. Trujillo. 1962. Adansonian classification of mycobacteria. J. Gen. Microbiol. 28:333-346.

4. Bönicke, R. 1966. The occurrence of atypical mycobacteria in the environment of man and animal. Bull. Int Union Tuberc. 37:361-368.

5. Bönicke, R., and J. L. Stottmeier. 1965. Erkennung und Identifizierung von Stämmen der Species Mycobacterium borstelense. Beitr. Klin. Erforsch. Tuberk. Lungenkr. 130:210-222.

6. Chamoiseau, G. 1979. Etiology of farcy in African bovines: nomenclature of the casual organisms Mycobac terium farcinogenes Chamoiseau and Mycobacterium senegalense (Chamoiseau) comb. nov. Int. J. Syst. Bacteriol. 29:407-410.

7. Da Costa Cruz, J. 1938. "Mycobacterium fortuitum." Um novo bacilo acido-resistente patogenico para o homen. Acta Med. (Rio de Janeiro) 1:297-301.

8. Gordon, R. E., and M. M. Smith. 1953. Rapidly growing, acid fast bacteria. I. Species descriptions of Mycobacterium phlei Lehmann and Neumann and Mycobacte. rium smegmatis (Trevisan) Lehmann and Neumann. J. Bacteriol. 66:41-48.

9. Gordon, R. E., and M. M. Smith. 1955. Rapidly growing, acid fast bacteria. II. Species description of Mycobacterium fortuitum Cruz. J. Bacteriol. 69:502-507.

10. Grange, J. M., and J. L. Stanford. 1974. Reevaluation of Mycobacterium fortuitum (synonym: Mycobacterium ranae). Int. J. Syst. Bacteriol. 24:320-329.

11. Haas, H., J. Michel, and T. Sacks. 1974. Identification of Mycobacterium fortuitum, Mycobacterium absces. sus, and Mycobacterium borstelense by polyacrylamide gel electrophoresis of their cell proteins. Int. J. Syst. Bacteriol. 24:366-369.

12. Jenkins, P. A., J. Marks, and W. B. Schaefer. 1971. Lipid chromatography and seroagglutination in the classification of rapidly growing mycobacteria. Am. Rev. Respir. Dis. 103:179-187.

13. Kubica, G. P., I. Baess, R. E. Gordon, P. A., Jenkins, J. B. G. Kwapinski, C. McDurmont, S. R. Pattyn, H. Saito, V. Silcox, J. L. Stanford, K. Takeya, and M. Tsukamura. 1972. A co-operative numerical analysis of rapidly growing mycobacteria. J. Gen. Microbiol. 73:55-70.

14. Lehmann, K. B., and R. O. Neumann. 1899. Lehmann's Medizinische Handatlanten, X. Atlas und Grundriss der Bakteriologie und Lehrbuch der speziellen bakteriologischen Diagnostik, vol. 2 (M. smegmatis, p. 403; $M$. phlei, p. 411). (Cited in R. E. Buchanan, J. G. Holt, and E. F. Lessel, Jr. [ed.], Index Bergeyana, The Williams \& Wilkins Co., Baltimore, 1966.)

15. Moore, M., and J. B. Frerichs. 1953. An unusual acid fast infection of the knee with subcutaneous abscesslike lesions of the gluteal region. J. Invest. Dermatol. 20:133-169.

16. Pattyn, S. R., M. Magnusson, J. L. Stanford, and J. M. Grange. 1974. A study of Mycobacterium fortuitum (ranae). J. Med. Microbiol. 7:67-76.

17. Runyon, E. H., L. G. Wayne, and G. P. Kubica. 1974. Family II. Mycobacteriaceae Chester 1897, 63, p. 681701. In R. E. Buchanan and N. E. Gibbons (ed.), Bergey's manual of determinative bacteriology, 8th ed. The Williams \& Wilkins Co., Baltimore.

18. Saito, H., R. E. Gordon, I. Juhlin, W. Käppler, J. B. G. Kwapinski, C. MeDurmont, S. R. Pattyn, E. H. 
Runyon, J. L. Stanford, I. Tarnok, H. Tasaka, M. Tsukamura, and J. Weiszfeiler. 1977. Cooperative numerical analysis of rapidly growing mycobacteria. The second report. Int. J. Syst. Bacteriol. 27:75-85.

19. Skerman, V. B. D., V. McGowan, and P. H. A. Sneath. 1980. Approved lists of bacterial names. Int. J. Syst. Bacteriol. 30:225-420.

20. Sokal, R. R., and P. H. A. Sneath. 1963. Principles of numerical taxonomy. W. H. Freeman, San Francisco.

21. Stanford, J. L., and A. Beck. 1969. Bacteriological and serological studies of fast growing mycobacteria identified as Mycobacterium friedmannii. J. Gen. Microbiol. 58:99-106.

22. Stanford, J. L., and J. M. Grange. 1974. The meaning and structure of species as applied to mycobacteria. Tubercle 55:143-152.

23. Stanford, J. L., W. J. Gunthorpe, S. R. Pattyn, and F. Portaels. 1972. Studies on Mycobacterium chelonei. J. Med. Microbiol. 5:171-182.

24. Takeya, K., Y. Nakayama, and H. Nakayama. 1967. Relationship between Mycobacterium fortuitum and Mycobacterium runyonii. Am. Rev. Respir. Dis. 96: 532-535.

25. Tsukamura, M. 1966. Adansonian classification of mycobacteria. J. Gen. Microbiol. 45:253-273.

26. Teukamura, M. 1967. Identification of mycobacteria. Tubercle 48:311-338.

27. Tsukamura, M. 1967. Mycobacterium chitae: a new species. Jpn. J. Microbiol. 11:43-47.

28. Tsukamura, M. 1970. Differentiation between Mycobacterium abscessus and Mycobacterium borstelense. Am. Rev. Respir. Dis. 101:426-428.

29. Tsukamura, M. 1971. Some considerations on classification of mycobacteria. Definition of bacterial species by introduction of the concept of "hypothetical median or mean organism." Jpn. J. Tuberc. Chest Dis. 17:18-30.

30. Tsukamura, M. 1972. Mycobacterium agri Tsukamura sp. nov. A new relatively thermophilic Mycobacterium. Med. Biol. 85:153-156. (In Japanese.)
31. Tsukamura, M. 1975. Identification of mycobacteria. National Chubu Hospital, Obu, Aichi, Japan.

32. Tsukamura, M. 1976. Mycobacterium agri: a new species. Actinomycetes Related Org. 11:156-157.

33. Tsukamura, M. 1976. Numerical classification of slowly growing mycobacteria. Int. J. Syst. Bacteriol. 26:409 420.

34. Tsukamura, M. 1976. Properties of Mycobacterium smegmatis freshly isolated from soil. Jpn. J. Microbiol. 20:355-356.

35. Tsukamura, M. 1977. Infections due to Group IV mycobacteria (a review). Iryo 31:1187-1196. (In Japanese and English.)

36. Tsukamura, M. 1977. Source and infection route to humans of pathogenic mycobacteria other than tubercle bacilli. Kekkaku 52:261-267. (In Japanese.)

37. Tsukamura, M., and S. Mizuno. 1968. "Hypothetical mean organisms" of mycobacteria. Jpn. J. Microbiol. 12:371-384

38. Tsukamura, M., S. Mizuno, and S. Tsukamura. 1968. Classification of rapidly growing mycobacteria. Jpn. J. Microbiol. 12:151-166.

39. Tsukamura, M., S. Mizuno, S. Tsukamura, and J. Tsukamura. 1979. Comprehensive numerical classification of 369 strains of Mycobacterium, Rhodococcus, and Nocardia. Int. J. Syst. Bacteriol. 29:110-129.

40. Tsukamura, M., S. Tsukamura, and S. Mizuno. 1967. Numerical taxonomy of Mycobacterium fortuitum. Jpn. J. Microbiol. 11:243-252.

41. Vanden Berghe, and S. R. Pattyn. 1979. Comparison of proteins from Mycobacterium fortuitum, Mycobacterium nonchromogenicum and Mycobacterium terrae using flat bed electrophoresis. J. Gen. Microbiol. 111: 283-291.

42. Weiszfeiler, J. G., V. Karasseva, and E. Karczag. 1969. Comparative studies on the taxonomic relationship between Mycobacterium abscessus and Mycobac terium borstelense. Acta Microbiol. Acad. Sci. Hung. 16:371-379. 